\title{
A Survey of Energy Efficient Unequal Clustering Algorithms for Wireless Sensor Networks
}

\author{
G. Vennira Selvi, \\ Research Scholar, \\ Department of Computer Science and Engineering, \\ Pondicherry Engineering College, Pondicherry.
}

\author{
R.Manoharan, \\ Professor, \\ Department of Computer Science and Engineering, \\ Pondicherry Engineering College, Pondicherry.
}

\begin{abstract}
In order to prolong the lifetime of sensor nodes, wireless sensor networks are partitioned into clusters. The most of the proposed Equal Clustering algorithms do not consider location of the base station. This situation causes hot spots or energy hole problem in multi-hop data forwarding. Unequal clustering mechanisms, which are designed by considering the location of the base station to solve the hot spot issue. In this paper, we examine currently proposed Unequal clustering algorithms for WSN. We will in brief discuss the operations of these algorithms, as well as the comparisons on the performance such as efficiency and quality between various schemes.
\end{abstract}

\section{General Terms}

Wireless Sensor Networks, Clustering.

\section{Keywords}

Clustering, Energy consumption, Network life time.

\section{INTRODUCTION}

Advance developments in micro-electro-mechanical systems (MEMS) and wireless communication have led to the introduction of sensor nodes which are smaller in size, low price and low memory power, communicating and computing device [1]. WSN (wireless Sensor network) consisting thousands of spatially distributed sensor nodes to monitor the changes in the military applications [2]. Due to limited energy constrains of sensor nodes, the improvement of energy efficiency of sensor networks is an important issue.

Earlier researchers focused on minimizing energy consumption of individual sensor nodes to attain energy efficiency whereas later researchers realized that minimizing overall energy resource of WSNs is more important to prolong network lifetime.

In order to increase energy efficiency and network lifetime, sensor nodes can be hierarchically grouped into clusters. In general, each cluster has a cluster head $(\mathrm{CH})$ which are responsible for gathering data and aggregating data from their cluster members [3]. Each cluster member forwards its data packets to the cluster-head, the cluster head aggregate and send it to the base station. The dissipation of energy in cluster head is higher than the cluster member when data is transmitted to the longer distance [4] [5]. The local changes need not be reflected to entire network, when cluster structure is used in WSN. This reduces the information processed by sensor nodes and data stored in sensor nodes

A fundamental issue in wireless sensor networks is maximizing the network lifetime subject to a given energy constraints. To achieve this goal, energy consumption must be balanced along with nodes. In homogeneous networks, the cluster head role can be periodically rotated among the sensor nodes to balance the energy dissipation. But, the hot spot problem cannot be avoided. The main objective of the reclustering is to balance the energy consumptions among the sensor nodes in each cluster, and it difficult to balance the energy consumption among cluster heads in the inter-cluster multihop routing development. We also suggest that using node's residual energy (RE) as the only criterion when selecting cluster heads is not sufficient to balance energy consumption across the network [9]. Selecting cluster head with higher residual energy can only be helpful to balance energy consumption among nodes within a cluster radius in the long term. It is not an effective to balance the load among different cluster heads to avoid the hot spot problem if the cluster heads are uniformly distributed among the network. Because sensor nodes in the hot spot still die prematurely, it cannot make efficient use of all nodes' energy. Unequal clustering algorithms are introduced to improve the network life time more efficiently and to mitigate the hot spot problem.

\section{UNEQUAL CLUSTERING ALGORITHMS FOR WSN}

The sensor nodes closer to the base station consume more energy, because the network traffic increases nearer base station [14]. Therefore, the nodes closer to the base station die prematurely. In order to balance energy consumption over the network, unequal clustering approach is introduced. In order to preserve more energy for inter-cluster data forwarding, the network is divided into clusters of unequal size and clusters closer to the base station have smaller size than those farther away from the base station. In this paper, we provide a complete survey of unequal clustering algorithm for wireless sensor networks. We also summarize and categorize the algorithms based on selecting cluster head and network lifetime. The most widely used unequal clustering algorithm has been chosen for comparison according to various properties is presented in Table 1 .

\subsection{Energy-Efficient Unequal Clustering (EEUC)}

[6] is a competitive based distributed unequal clustering algorithm where cluster-heads are elected based on the high residual energy of its neighbor and its distance to the base station (BS). To address the hot spot problem, EEUC divide the nodes into the cluster with unequal size, and cluster closer to the base station have smaller sizes that those father away from the BS because the node could not communicate directly to the BS due to the limited transmission range. Each node is assigned by competitive range. This competitive range decreases distance to the base station. This result is that the clusters closer to the base station are smaller in size thus the $\mathrm{CH}$ will consume less energy during intra-cluster communication and can preserve more energy for inter-cluster communication. EEUC algorithm is also a probabilistic 
clustering algorithm, because in each cluster creation round, each node generates a random number between 0 and 1 to decide whether it is going to participate to the cluster-head election or not. If a sensor node has decided to join to the cluster head election, then it becomes a tentative cluster head. Tentative cluster head in local regions participate in order to become an actual cluster head. This competition is based on the residual energy of each tentative cluster-head. After cluster-head election is done, the remaining sensor nodes join to the closest cluster.

\subsection{Multihop Routing Protocol with Unequal Clustering (MRPUC)}

[11] elects the $\mathrm{CH}$ in rounds with high residual energy. There are three phases such as Cluster setup, Inter-cluster multihop routing formation, and Data transmission for each round. The data transmission phase takes more duration compare than other two phases in order to minimize the overhead of sensor nodes. Initially, every nodes in the sensor networks has the responsibility to collect the information from its neighbors by broadcasting a HELLO message to its neighbors. Initially all the nodes are in unknown status. The node is elected as a $\mathrm{CH}$, if it has the highest residual energy among all neighbor nodes and broadcast HEAD-MSG to all nearest node to build a cluster. Depending upon the received signal strength, each neighbor nodes computes the distance $d(i, j)$ to the BS based on the competitive radius. If a node is not fit into the radius of any $\mathrm{CH}$, and all its neighbors with larger residual energy than it have joined with other clusters, then the node is elected as $\mathrm{CH}$ passively. To mitigate the hot spot problem, the MRPU assume the multihop data transmission and construct an intercluster tree rooted at Bs to save energy. The minimum cost node is considered as a parent node among all neighbors $\mathrm{CHs}$. The data transmission begins when the inter-cluster tree is constructed and each node broadcast the sensing data to the $\mathrm{CH}$ during its allocated transmission time. The $\mathrm{CH}$ gathers data packets into a single packet and sends data to the parent node which forwards the received packet toward BS. The next round is begins after a certain time which is determined in advance. MRPUC outperforms an equal clustered version of itself by extending the network lifetime by $34.4 \%$.

\subsection{Unequal Hierarchical Energy Efficient Distributed Clustering (UHEED)}

[12] is an unequal clustering algorithm, extended from HEED. The unequal size is created based on the distance of the $\mathrm{CH}$ from the BS. The formula competition radius from EEUC [6] is used to create a smaller cluster nearer to the BS. The amount of intra-cluster traffic is considerably reduced nearer to the BS, by creating unequal sized clusters. UHEED make the following assumptions about node : (i) all nodes are homogeneous in terms of energy, communication and processing capabilities;(ii) each node is identified with a unique ID; (iii) nodes can transmit at various power levels depending on the distance of the receivers; (iv) nodes are not mobile that is they remain stationary after the uniformly distributed deployment process; (v) communicating nodes can establish the distance among them1; (vi) all nodes know their distance from the base station. The BS is located away from the sensing grid with no energy concerns at all, and it is considered to be a node with enhanced communication and computation capabilities. The BS is not portable. The data captured in a cluster is highly correlated; therefore it can be aggregated before being transmitted to the base station. The hot spot problem is effectively mitigated in UHEED than equal sized clusters and balances the energy consumption among the sensor nodes in the network.

\subsection{Energy Efficient Distributed Unequal Clustering (EEDUC)}

[13] is a distributed unequal clustering algorithm, in which the cluster head can be distributed by using waiting time. The waiting time is measured with the parameters of residual energy, number of neighborhood node. Each node broadcast the advertisement message to count the number of neighborhood nodes (NN) in 1-hop range to calculate the distance from the base station. Base station assigns the value for each node to decide a time unit for cluster formation. EEDUC consider the number of neighborhood node of node $i$ to cover the whole network. The waiting time of each sensor node is synchronizing with the node time. When it reaches 0 , a node determined itself to be $\mathrm{CH}$. The $\mathrm{CH}$ broadcast a HELLO message to neighbor nodes. The competition radius of HELLO message is determined by the distance to the BS, the number of neighbor nodes and the residual energy of $\mathrm{CH}$. Neighborhood node stores the $\mathrm{CH}$ formation in their node table and changes its mode as a cluster member. The member mode sends a reply message about their information to the $\mathrm{CH}$. When re-clustering to the next round, the waiting time is decided by each node in the networks. When compared with EEUC, the energy consumption of EEDUC is improved up to $24.2 \%$.

\subsection{An Energy-Driven Unequal Clustering (EDUC)}

[10] In EDUC, nodes use uneven competition ranges to construct clusters of uneven sizes. Clusters farther away from the BS have smaller sizes in order to preserve some energy for long-distance data transmission. Therefore, the energy consumption among cluster heads is balanced effectively. The cluster head can be rotated based on the energy level of cluster head to minimizeize the unnecessary energy waste. Each node acts as cluster head no more than once during the whole network lifetime. Thus, EDUC minimizes the additional cost and achieves high energy efficiency. In this paper, the energy level is computed accurately in cluster head rotation based on the assumption that the cluster head is a single-hop communication from the BS. However, the single-hop assumption may not always be appropriate for some real situation. In clustering multihop networks using a random competition scheme for electing cluster head, the number of packages forwarded by a cluster head is not easy to estimate while computing the energy threshold. Therefore, the energydriven cluster head rotation scheme proposed here is not suitable for multihop networks, because the energy level is assigned to be very precise.

\subsection{An Unequal Cluster-based Routing (UCR)}

[8] to mitigate the hot spot problem, the nodes are grouped into unequal clusters. It is designed for inter-cluster relay traffic, which consists of two parts, one is EEUC to mitigate the hot spot problem and the other is a routing protocol for inter-cluster relay traffic [6]. In UCS, the energy consumption is maintained uniformly among all $\mathrm{CH}$ for long run by decreasing the number of nodes in cluster with high relay load near the base station. Initially, tentative $\mathrm{CHs}$ are randomly selected to compete for final $\mathrm{CH}$. Each tentative cluster head has a competition range. Different competition ranges are used to produce cluster of unequal size. Finally, one $\mathrm{CH}$ is allowed in each competition range. After a $\mathrm{CH}$ has been 
selected, each $\mathrm{CH}$ broadcast advertisement message across the network. Each node chooses its nearby $\mathrm{CH}$ with largest received signal strength and sending joining cluster message to the nearest cluster head. Then the voronoi of sensor node is constructed. UCR assumes that the approximate distance from one sensor to another based on the received signal strength. In real environment, error will arise due to the noise.

\subsection{Unequal Clustering Size (UCS)}

[7] An unequal clustering model was first proposed in (Soro and Heinzelman, 2005) based on unequal clustering size (UCS) to balance the energy consumption of cluster heads due to heavy inter-cluster relay traffic. In UCS, N nodes are randomly distributed over a circular area of radius R. BS is located at the centre of observed area and it receives all collected information from the $\mathrm{CH}$. The data forwarding can be done through multihops. Each $\mathrm{CH}$ chooses a closest $\mathrm{CH}$ to forward the aggregated data to base station. In general, $\mathrm{CH}$ closer to the base station creates unbalanced energy consumption among the all the CHs. It maintains more uniform energy consumption among the $\mathrm{CH}$ by rotating the cluster head in every cluster. In UCS, the $\mathrm{CH}$ positions are determined in before to arrange the $\mathrm{CHs}$ in symmetrically concentric circle around the base station. Each cluster nodes are collected in the voronoi region around the $\mathrm{CH}$. This presents a layered network in which every layer contains a particular number of clusters. UCS assumes that all clusters in one layer has the same size and shape but it different from one another. In multi-hop networks, UCS was $10-30 \%$ better than existing equal clustering models.

Table 1. Comparison of unequal clustering algorithms for WSN

\begin{tabular}{|c|c|c|c|c|c|c|c|c|c|c|c|c|c|}
\hline \multirow[t]{2}{*}{$\begin{array}{l}\text { Clustering } \\
\text { Algorithm }\end{array}$} & \multicolumn{3}{|c|}{$\begin{array}{c}\text { Parameters } \\
\text { used to select } \\
\mathrm{CH}\end{array}$} & \multicolumn{2}{|c|}{$\begin{array}{c}\text { Connectiv } \\
\text { ity of } \mathrm{CH} \\
\text { to } \mathrm{BS}\end{array}$} & \multirow[t]{2}{*}{$\begin{array}{c}\text { Node } \\
\text { Deployment in } \\
\text { fields }\end{array}$} & \multirow[t]{2}{*}{$\begin{array}{l}\text { Node } \\
\text { type }\end{array}$} & \multirow[t]{2}{*}{$\begin{array}{c}\text { Mode of } \mathrm{CH} \\
\text { Selection }\end{array}$} & \multirow{2}{*}{$\begin{array}{l}\text { Number } \\
\text { of Nodes } \\
\text { in cluster }\end{array}$} & \multirow[t]{2}{*}{$\begin{array}{c}\text { Energy } \\
\text { Efficiency }\end{array}$} & \multirow[t]{2}{*}{$\begin{array}{c}\text { Balanced } \\
\text { Cluster }\end{array}$} & \multirow[t]{2}{*}{$\begin{array}{l}\text { BS Location } \\
\text { Awareness }\end{array}$} & \multirow[t]{2}{*}{$\begin{array}{c}\text { Heterogeneity } \\
\text { Level }\end{array}$} \\
\hline & RE & BS & NN & $\mathrm{SH}$ & $\mathrm{MH}$ & & & & & & & & \\
\hline$M R P U C$ & $\checkmark$ & $x$ & $\checkmark$ & $x$ & $\checkmark$ & Square area & Homo & Neighbor & Unequal & Moderate & No & $x$ & Multi \\
\hline$E E U C$ & $\checkmark$ & $x$ & $x$ & $x$ & $\checkmark$ & Square area & Homo & Probabilistic & Unequal & Good & Yes & $x$ & Multi \\
\hline$E E D U C$ & $\checkmark$ & $x$ & $\checkmark$ & $\checkmark$ & $\checkmark$ & Square area & Hetero & Probabilistic & Unequal & Moderate & No & $x$ & Multi \\
\hline$U C S$ & $\checkmark$ & $x$ & $x$ & $x$ & $\checkmark$ & Circular area & Homo & Probabilistic & Equal & Good & Yes & $\checkmark$ & Two \\
\hline$U C R$ & $\checkmark$ & $x$ & $x$ & $x$ & $\checkmark$ & Circular area & Homo & Probabilistic & Equal & Good & Yes & $x$ & Tow \\
\hline$E D U C$ & $\checkmark$ & $x$ & $x$ & $x$ & $\checkmark$ & Square area & Hetero & Probabilistic & Unequal & Moderate & No & $x$ & Multi \\
\hline UHEED & $\checkmark$ & $x$ & $x$ & $x$ & $\checkmark$ & Square area & Homo & Probabilistic & Unequal & Moderate & No & $x$ & Multi \\
\hline
\end{tabular}

Homo: Homogeneous; Hetero: Heterogeneous; Multi: Multi Level ; Two: Two Level

\section{ONCLUSION AND FUTURE WORK}

In this paper we presented the detail survey of proposed unequal cluster algorithms, specifically with respect to their cluster size, energy consumption and data forwarding. In WSN, the energy limitations of node play a vital role in designing any protocol implementations. From our surveyed work, all proposed algorithms are energy-aware routing protocol for WSN to mitigate the hot spot problem by choosing the clusters that are closer to the base station have smaller size and balance the energy consumption among all nodes. We classified all protocols according to cluster head selection, energy consumption, inter-cluster, and intra-cluster communication, stability and location awareness of sensor nodes. All the above existing algorithms are selecting a $\mathrm{CH}$ with node's residual energy. We suggest that, it is not sufficient to balance the energy consumption for multihop data forwarding. In future, we propose to select a $\mathrm{CH}$ with more residual energy instead of selecting nearest neighborhood $\mathrm{CH}$. 


\section{REFERENCES}

[1] I. F. Akyildiz, W. Su, Y. Sankarasubramaniam, and E. Cayirci, "A Survey on Sensor Networks", IEEE Communications Magazine, vol. 40, no. 8, pp. 102-114, 2002.

[2] O. Younis and S. Fahmy, "HEED: a hybrid,energyefficient, distributed clustering approach for Ad hoc sensor networks," IEEE Transactions onMobile Computing, vol. 3, no. 4, pp. 366-379, 2004.

[3] Yick J, Mukherjee B, Ghosal D.Wireless sensor network survey. Computer Networks 2008; 52(12): 2292--2330.

[4] Younis O, Fahmy S. Distributed clustering in ad hoc sensor networks: a hybrid, energy-efficient approach. Proceedings of IEEEINFOCOM, 2004; an extended version appeared in IEEE Transaction Mobile Computing, vol. 3, no. 4, 2004; 366--379.

[5] Ahmed AA, Mohammed Y. A survey on clusterin algorithms for wireless sensor networks. Elsevier, Computer Communications 2007; 30: 2826--2841.

[6] C. F. Li, M. Ye, G. H. Chen, and J. Wu, "An energyefficient unequal clustering mechanism for wireless sensor networks," in Proceedings of the IEEE International Conference on Mobile Adhoc and Sensor Systems Conference, pp. 1-8, IEEE Press, Washington, DC, USA, November.

[7] Soro S, Heinzelman WB. Prolonging the lifetime of wireless sensor networks via unequal clustering. Proceedings ofParallel and Distributed Processing Symposium, 2005.
[8] Chen G, Li C, Ye M, Wu J. An unequal cluster-based routing protocol in wireless sensor networks. Wireless Networks Journal 2009; 15(2): 193--207.

[9] Yang J, Zhang D. An energy-balancing unequal clustering protocol for wireless sensor networks. Information Technology Journal 2009; 8: 57--63.

[10] Yu, J., Qi, Y., and Wang, G. (2011). An energy-driven unequal clustering protocol for heterogeneous wireless sensor networks. Journal of Control Theory and Applications, 30(12):133-139

[11] Bencan Gong, Layuan Li, Shaorong Wang, Xuejun Zhou. Multihop Routing Protocol with Unequal Clustering for Wireless sensor Networks. IEEE,2008,pp552-556

[12] Ever, R. Luchmun, L. Mostarda, A. Navarra and P. Shah, (2012), "UHEED - an Unequal Clustering Algorithm for Wireless Sensor Networks", In Proceedings of the 1st International conference on sensor networks (SENSORNETS 2012). Rome, Italy February 24-26. 185-193

[13] Sungju Lee, Jangsoo Lee , Hongjoong Sin, Seunghwan Yoo, Sanghyuck Lee, Jaesik Lee, Yongjun Lee, and Sungchun Kim. An Energy-Efficient Distributed Unequal Clustering Protocol for Wireless Sensor Networks, World Academy of Science, Engineering and Technology 482008

[14] M. Ye, C. F. Li, G. H. Chen, and J. Wu, "EECS: An Energy Efficient Clustering Scheme in Wireless Sensor Networks", in Proceedings of IEEE Int'l Performance Computing and Communications Conference (IPCCC), 2005, pp. 535-54 